\title{
An approach for Risk Assessment Score of Suicide Attempt (RASSA)
}

\author{
Ana Fructuoso ( $\nabla$ afrucas@gmail.com ) \\ University of Valladolid \\ Inmaculada Fierro \\ University of Valladolid \\ María-Isabel Jiménez-Serranía \\ University of Valladolid \\ Alfonso Carvajal Garcia-Pando \\ University of Valladolid
}

\section{Research Article}

Keywords: suicide attempt risk, antipsychotics, antidepressants, opioids, non-psychiatric comorbidity.

Posted Date: February 1st, 2021

DOI: https://doi.org/10.21203/rs.3.rs-191353/v1

License: (9) This work is licensed under a Creative Commons Attribution 4.0 International License. Read Full License 


\section{Abstract}

Background: Suicide remains a leading cause of death and psychiatric population is often at increased risk for suicide. Therefore, there is a persistent need for well-designed clinical instruments that allows us to identify relevant risk factors. Our study aims to improve patient follow-up and identify possible suicide risk markers from a passage to self-harm among hospitalized psychiatric patients.

Methods: This case-control study included the review of psychiatric, sociodemographic, drug use, and other health-related data, retrieved from 1,680 psychiatric patients' health records. Differences between comparative groups were examined, and stepwise logistic regression analyses were performed to identify suicide risk factors within this population.

Results: From the analysis of 560 suicide attempters' clinical records, thirteen risk items were included in our final model, named as Risk Assessment Score of Suicide Attempt (RASSA). The factors that scored the highest in this model were 'not taking antipsychotic medication', 'somatic comorbidity', and 'a family history of suicide'. Suffering from depression has a high score, and treatment with selective serotonin reuptake inhibitors (SSRIs) is also involved in the risk of a suicide attempt. Regarding medication use, opioid analgesics decreased the risk score, while taking non-opioid analgesics increased it. In terms of commonly abused substances, alcohol, cocaine, and amphetamine dependence increased the score. A higher risk was also associated with cannabis dependence, while tobacco use reduced it. As for demographics, the risk was significantly greater for women and subjects who were unmarried.

Conclusions: The proposed model of risk assessment score of suicide attempt (RASSA) offers the possibility of establishing a suicide attempt risk based on data directly gathered from the health records of psychiatric patients. Therefore, it might be used as an initial screening test before patient evaluation and psychometric tests.

\section{Background}

Suicide risk assessment scales are designed to predict the risk of future-self harm and suicide attempt [13]. For example, Beck's Suicide Intent Scale (SIS) and Beck's Scale for Suicide Ideation (BSI) are widely used in research and clinical practice. Despite the value of these scales in the assessment of suicidal risk, they require the patient's cooperation and prior medical intervention, limiting their scope of application. Yet suicide remains a leading cause of death, and there is a persistent need for well-designed clinical instruments that allow us to make progress in establishing risk factors [4] and improving suicide prediction [5]. To this end, new and creative approaches are required [6]. Using health records systems to distinguish higher from lower-risk individuals or to readily access prior treatment [7] may allow for an earlier stage of intervention [8], from either primary care settings or psychiatric institutions. This can ultimately lead to the goal of reaching sooner the higher risk individuals [9].

Risk factors for attempting suicide are diverse and interrelated in complex ways. Associations have been found between suicide and most psychiatric disorders, such as psychosis and schizophrenia [10], major 
depression, dysthymia, and bipolar depression [11-13]. Research appears to indicate that the risk of developing suicidal adverse drug reactions differs according to drug types [10,14]. In this sense, suicidal risk is notably increased by selective serotonin reuptake inhibitors (SSRIs antidepressants), whose net effect on suicidality furthermore appears to be dependent on the patient's age [14-17]. Likewise, suicide has been related to drugs used in treatments of non-psychiatric, chronic, and painful diseases [18-20]. In addition to the more readily apparent risk factors, a variety of demographic, cognitive and psychosocial factors present significant implications with suicide attempt risk, such as gender, marital status [21], family psychopathology [9] and abuse, negative life events, hopelessness, and problems specific to the various stages of human development. Moreover, there is evidence of a genetic component to suicide risk because of its overlap with major depression [22].

Psychiatric population is known to be often at increased risk for suicide, so there is always a need to identify factors pertinent to such risk, in order to develop strategies aimed at mitigating it. However, the scarce of studies for some risk factors, especially for suicide attempt and suicidal ideation, leaves gaps in knowledge that still need to be properly filled up. In this work, we present an exploration of independent factors associated with suicide risk among psychiatric patients. We propose the use of medical records to identify suicide score markers in patients who have or not previously made a suicide attempt. Our study can provide new evidence and guide future lines of research based on the data collected in medical records.

\section{Methods}

\section{Subjects}

All participants in the study were subjects who could be assessed retrospectively using structured questionnaires for gathering information from their health records. The subjects were patients between the ages of 18 and 97 who had been admitted to the Brief Hospitalization Unit of Psychiatry at the Complejo Asistencial Universitario de Palencia- Hospital San Telmo (Palencia, Spain) between January 2000 and January 2014. Criteria for inclusion into the case group was having a suicide attempt; that is, an act of self-injury carried out intending to end the own life at least once. When pertinent, the last episode was the one retained for our analysis. For each case, two control subjects (which did not commit suicide attempt) were selected. The control group comprised patients who had been admitted into the psychiatric unit around the same period (within 3 months of the case group patient's admission date) for any reason (most often for the treatment of a mood disorder), except attempted suicide. The matching was based on age (the closest match within 0 years and the furthest match within 5 years), gender and date of admission into the hospitalization unit (within 3 months of the case group patient's admission date). Exclusion criteria for both groups were being under 18 years of age and patients whose medication prescription record was not registered in the three months prior to hospital admission. The study protocol was approved (CIB-005:20114006) by the Clinical Research Committee at Complejo Asistencial Universitario of Palencia on November $4^{\text {th }}, 2014$. 


\section{Measures}

Data was extracted retrospectively from paper and computerized medical record systems, which included hospital admission and discharge data. Hospital admissions were from the emergency department (77\%) or other medical units $(23 \%)$. For each subject, we first identified three sociodemographic variables: age, sex, and marital status. We proceeded to identify variables relating to the suicidal behaviour and clinical severity. These variables were: psychiatric history according to DSM-5 (Diagnostic and Statistical Manual of Mental Disorders. Fifth Edition, 2018) criteria, number of previous suicide attempts, family history of suicide (No/Yes), and number of visits to the emergency department prior to the last suicide attempt. Next, we identified personality traits according to MCMI-IV (Millon Clinical Multiaxial Inventory, Fourth Edition, 2015), selecting the feature with the highest score. We recorded the presence of depression as the main diagnosis (No/Yes), as defined by DSM-5, and any episode of major depression in the three months prior to the suicide attempt (No/Yes). Main somatic diagnosis (non-psychiatric comorbidity) under ICD-11 (International Classification of Diseases, Eleventh Revision, 2019) criteria was also recorded. Attendance/non-attendance to a psychotherapy program were also identified in the medical history.

We further classified patients according to exposure or non-exposure to the following prescription drugs in the three months prior to the hospital admission: anticonvulsants, antidepressants, antipsychotics, anxiolytics (benzodiazepines), and analgesics (opioids and non-opioids). In addition, since patients who had been diagnosed with a substance use disorder also benefitted from care by the Addiction Care department, we identified substance use disorders from the patients' records to any of the following substances in the three months prior to the suicide attempt: alcohol, tobacco, cocaine, cannabis, heroin, and amphetamines. This allowed us to determine dependence levels according to DSM-5 and ICD-11 criteria, and patients were then classified into one of two categories: 1) Substance abusers, for those suffering from severe ( $>6$ criteria) and moderate dependence (4-5 criteria) and 2) Sporadic consumers (< 3 criteria).

\section{Statistical Analysis}

Categorical variables were recorded with absolute and relative frequencies (percentages), and continuous ones (non-normally distributed) with median and interquartile ranges (IQR = Q3-Q1). Differences between the case and control groups were examined using U Mann-Whitney test for continuous variables and Pearson $\chi^{2}$ test for categorical data. Stepwise logistic regression analyses were performed to identify factors associated with risk of suicide attempt. Odds ratios (OR) and $95 \%$ confidence intervals $(95 \% \mathrm{Cl})$ are reported. Independent variables introduced in the models were carefully selected to avoid confounding effects, and the possible multicollinearity of the model was checked with an analysis of tolerance and inflation statistics [23]. To adjust the coefficients with greater precision -to avoid losing cases with the rest of the variables that were not significant-, the logistic regression was repeated with the variables that had been significant in the previous analysis. A multivariate logistic regression model was developed, and internal validation processes were performed using bootstrapping random sampling 
with 1,000-fold cross-validation. This logistic regression model was tested for calibration with a HosmerLemeshow $\chi^{2}$ test. To measure the discriminating accuracy of the model and individual items, the area under the curve (AUC) and its $95 \% \mathrm{Cl}$ of the receiver-operating characteristic (ROC) were scrutinized. The above model was subsequently translated into a risk score for suicide attempt, developed on the respective weights of the significant variables in the model (based on their regression coefficients). Each individual score was yielded via a linear transformation of the corresponding regression coefficient ( $\beta i)$, which was divided by the lowest $\beta$ i value, multiplied by the constant, and then rounded to the nearest integer. The overall score of any given patient was the sum of all individual scores. The proportion of cases with previous suicide attempts was considered to adjust the final score of the subjects that had a previous suicide attempt. To measure the discriminating accuracy of the total and individual scores, the area under ROC curves and its $95 \% \mathrm{Cl}$ were scrutinized again. From the ROC analysis, the optimal cut-off value for final score was identified at the point where the sum of sensitivity and specificity was the highest according to Youden's index (Youden's J). Sensitivity, specificity, and positive and negative predictive values (PPV and NPV) for the optimal cut-off value were calculated. Statistical significance was defined as $p<0.05$ (two-tailed) for all tests. All statistical procedures were performed with SPSS 24.0 software.

\section{Results}

\section{Patients' demographic and characteristics}

After reviewing hospital admission records from January 2000 to 2014, and applying the aforementioned inclusion and exclusion criteria, we obtained a sample size of 1,680 patients: 560 suicide attempt cases and 1,120 controls. Two-third of cases were women, and half of the total were single. The median age was 34 years for both suicide attempt cases and control subjects. Females were younger than males (Table 1). Half of the suicide attempt cases had been diagnosed with depression as principal disorder. Diagnosis of depression was significantly more frequent among suicide attempt cases than control subjects. Furthermore, $47 \%$ of the cases had suffered a major depression episode (MDE-3) in the three months prior to the suicide attempt (Table 1). Personality traits were determined for $100 \%$ of case patients, but only for $9 \%$ of controls. Borderline personality and dependent personality traits $(64 \%$ and $15 \%$, respectively) were found in highest proportion among case subjects. Less than one-third of case and control patients were attending a psychotherapy program (Table 1). Proportion of comorbid somatic disease was higher among case patients ( $88 \%$ ) than controls (58\%, Table 1). Among the case patients, the most common disorders were musculoskeletal (45\%) and neurological (24\%), although gastrointestinal and oncological disorders were also identified.

Antidepressants, benzodiazepines, and non-opioid analgesics were the most commonly used medications in the case group (Table 1 and Table 2), followed by antipsychotics, anticonvulsants, and opioid analgesics. Most antidepressants belonged to the group of SSRIs (Table 2). The use of SSRIs among suicide attempt cases was prominent: $54 \%$ compared to $37 \%$ of controls (Table 1 ). Among the 
SSRIs antidepressants, $12 \%$ of case patients were treated with escitalopram, and 6 to $8 \%$ were treated with venlafaxine, paroxetine, and sertraline.

The use of non-opioids analgesics was also significantly higher among case subjects (Table 1). In contrast, antipsychotics were used significantly less frequently in the case group than in the control group. A substantial amount of control patients (74\%) had a history of substance use disorders, with significantly more addictions $\left(X^{2}=39.86\right.$, d.f. $\left.=1 ; p<0.0001\right)$. Still, more than one-half of case patients suffered from substance use disorders as well (Table 1). Alcohol, cocaine, and amphetamine dependence was significantly higher than in the control group. However, tobacco dependence was significantly lower (45\% vs. $59 \%$ in control group). We did not observe significant differences between groups in the frequency of cannabis (around 12\%) or heroin (lower than a 1\%) addiction.

Among case patients, 134 (24\%) had a family history of suicide. This variable was significantly associated with a suicide attempt (Table 1). Not surprisingly, a history of previous suicide attempts was also a factor significantly associated with a suicide attempt. Indeed, more than half of case patients had multiple (up to six recorded) suicide attempts. Among those subjects admitted at the hospital's emergency department (HED) in the three months prior to hospital admission, the median number of HED visits was higher among control subjects (Median $=2$; [Q1-Q3] = [1-2]) than among case patients (Median $=1$ [1-1]; U Mann-Whitney $=38721.5 ; p<0.0001)$.

\section{Risk factors Associated with Suicide Attempt among psychiatric patients}

The final logistic regression model for predicting risk of suicide attempt included thirteen risk factors that strongly increase, in a statistically significant manner, the predisposition to commit suicide. The model, after bootstrapping (1,000 random samples) for internal validation, is displayed in Table 3 . Factors that strongly increased the risk of suicide attempt from high to low OR were 'not using antipsychotic medication', 'comorbid non-psychiatric disorders', 'positive family history of suicide', 'use of non-opioid analgesics', 'major depression (as principal psychiatric disorder)', 'cocaine addiction', 'use of opioid analgesics', 'cannabis addiction', 'single (marital status)', 'an episode of major depression in the three months prior to the last suicide attempt', 'female (sex)', 'non-tobacco addiction', and 'use of SSRIs antidepressants' (Table 3). The final model was well calibrated (Hosmer-Lemeshow $X^{2}=7.04 ; d . f .=8 ; p=$ 0.532 ), and the area under the ROC curve was of 0.877 .

\section{RASSA: Risk Assessment Score of Suicide Attempt in psychiatric patients by retrospective analysis of health records}

RASSA scores are displayed in Figure 1. The overall score of a patient was the sum of all individual scores, adjusted by the factor of previous suicide attempt contribution to risk, considering the ratio of case patients with and without previous suicide attempts (327/233). Thus, we multiplied the final score of patients with a previous suicide attempt by 1.4. With this factor, the AUC of the ROC increased up to 0.929 (Figure 1). Optimal cut-off point (Youden's $\mathrm{J}=0.71$ ) was 23 points (Sensitivity $=0.83$; Specificity $=$ 
0.89; PPV $=0.79 ; \mathrm{NPV}=0.91$ ). Performance characteristics of RASSA at various cut-off points for evaluation of risk of suicide attempt score are presented in Table 4.

\section{Discussion}

Our results confirm that, by using the data commonly available in health information systems, it is possible to develop a tool for the screening of factors associated with suicide risk among psychiatric patients that does not require psychometric testing. This would be of great help to develop strategies aimed at mitigating such risk. The question is particularly relevant in light of the increasingly widespread adoption of electronic health records, which might provide opportunities for practical application of precision medicine and the possibility of predicting risky suicidal behaviour [6, 24]. The score model obtained (RASSA) is robust, with high sensitivity and specificity, and it will allow to identify the potential risk factor for suicide in a given individual. According to our model, the strongest prediction factors for identifying risk to commit suicide were 'not using antipsychotic medication', 'non-psychiatric comorbidity' -mainly skeletal muscle and neurological disorders, but also gastrointestinal and oncological disorders - , and 'a family history of suicide'.

Suicide behaviour is complex. Indeed, it has been shown that a variety of clinical features beyond mental health diagnoses appear among the top 100 predictors [24]. Numerous studies have identified pain as a risk factor for suicide, and it is estimated that up to $50 \%$ of patients with chronic pain present suicidal ideation [25]. Furthermore, pain can mask underlying depression [26]. The high-risk score of the family history of suicide variable agrees with evidence of the genetic influence on suicidal behaviour and its overlapping with major depression [22]. As expected, suicidal behaviour was associated with depression as the principal mental health diagnosis [27, 28,]. However, it is interesting to point out that RASSA suggests that suicide attempt risk can be high even in the absence of depression if other risk factors are present, and that depression alone could be not enough to explain a suicide attempt. In fact, if depression is the only risk factor observed in the patient (of the 13 factors considered), the risk obtained is low.

Case group subjects in our sample were more exposed to antidepressants than control subjects (74\% vs. $65.2 \%$ ), particularly to SSRIs ( $54 \%$ vs. $37 \%$ ). Frequency of case patients was higher in those exposed to escitalopram, venlafaxine, paroxetine, sertraline, citalopram, and fluvoxamine, which is consistent with previous results [15]. Thus, SSRIs appeared as a significant predictor of increased suicide risk in the univariate analysis and in the adjusted model. There was also an association between suicide attempt and use of certain medications. The protective effect of opioids, which appears after adjustment, is consistent with their well-known anxiolytic and sedative effects. Although mediated by different routes, this may be a similarly real effect to that of ketamine [29]. More difficult to explain is the association of non-opioid analgesics with the risk of a suicide attempt. This should be interpreted not as these analgesics provoke suicidal ideation, but that they might be a marker for those patients in high risk. Taking pills or pharmaceutical overdose is a common method of suicide attempt. NSAIDs and acetaminophen are widely used analgesics in the prescription and non-prescription settings and the literature shows that access to methods of suicide may increase the risk of it [30]. We found a protective 
factor related with antipsychotic drug use, but the contribution of antipsychotic drugs to suicide risk remains unclear. Whereas some observations suggest that the side effects of antipsychotic medications may contribute to suicidality, lack of adherence to antipsychotic drugs may increase suicide risk $[12,16$, 31].

Substance abuse was also associated with the risk of suicide attempt, since alcohol, cocaine, and amphetamine dependence were higher among case subjects. Alcohol dependence, alone or in combination with comorbid psychopathology or negative life events, is known to dramatically increase the risk of suicidal ideation, suicide attempts and completed suicides in the long term (see review in [16]). In explaining suicidal behaviour, one must account for the stimulus necessary to carry out the act, which could certainly be produced by cocaine and amphetamines. This explanation coincides with one that has been given to understand the possible action of SSRIs on suicidal behaviour [32], as SSRIs and cocaine share a similar mechanism in the post-ganglionic nerve termination, that is, the inhibition of neurotransmitter reuptake. Cannabis had a similar prevalence of use among case subjects and control subjects, but in the estimation of the adjusted risk, a higher risk of suicide attempt was found among those suffering from a cannabis addiction. This increased risk may be explained by the mood and personality deterioration associated with chronic use [33]. On the other hand, results about the relationship of tobacco or nicotine with suicidal behaviour are contradictory; while some have identified a positive association [34], independently of comorbid mental disorders and physical diseases [35], others have not found it $[36,37]$. These discrepancies could be due to the type of samples studied or to other associated factors, as smoking is particularly frequent among psychiatric patients, and even more so among patients who are medicated with antipsychotics. If antipsychotics exert a protective action on suicidal behaviour and these are associated to a greater extent with smoking [38, 39], the distribution found of this medication between case subjects $(21 \%)$ and control subjects $(31 \%)$ in our study would be consistent with this fact.

Finally, we also found that the risk of suicide attempt was significantly greater for women and for unmarried subjects. Although suicide rates are known to be higher among men, this is known to result from the more lethal suicide methods chosen by males. Indeed, the suicide attempt worldwide is higher in women than in men. This is a complex and multifactorial phenomenon. There is a multitude of potential social-cultural reasons for why the suicide attempt rate is higher among women, like their grater longevity. Another factor might be that women are more prone to depression than men [40]. However, mental disorders such as depression and schizophrenia are identified with suicidal behaviour in both men and women. Eating disorders are also correlated with an increased risk of suicide in women. As we know, the existence of possible violence, child abuse and factors related to pregnancy have also been associated with an increased risk of suicide in women [41]. Another possible explanation is the vulnerability related to gender and certain psychiatric pathologies, more prevalent in the case of women, as well as the existence of psychosocial stressors [42]. Hormonal disturbances, such as changes in the oestradiol level that occur during the menstrual cycle, may also be related to the higher frequency of suicides among women [43]. Nevertheless, this question remains to be fully dilucidated. On the other hand, the greater risk of suicide attempt for people who were unmarried may be explained by the risk for depression caused by 
isolation and thus for potential suicidal behaviour, which agrees with our results. Similarly, it has been shown that for both men and women, "separated marital status" is associated with more than a fourfold risk of suicidal behaviour compared with married patients [24].

Like all observational studies, the present work has some obvious limitations. We cannot completely rule out the possible existence of biases or possible residual confounding factors due to variables that were not considered into evaluation. For instance, alcohol abuse is a risk factor for suicide and suicide attempt repeatedly noted in the literature [16]. In our study, alcohol abuse resulted as a significant variable for

suicide attempt in the univariate analysis but turned up not enough significant in the multivariate logistic regression after controlling for other factors. The alcohol abuse/dependence variable was strongly associated with the rest of the abuse/dependence variables whose effects on suicide attempt risk are considered into score. As a matter of fact, the combined consumption of alcohol and tobacco has been reported higher risk of attempt of suicide than each substance separately [34]. For this reason, RASSA could underestimate the risk of suicide attempt in patients with no other diagnosis of abuse/dependency but alcohol. However, it must be said that it is not easy to gather as many cases as the ones presented in our study, but we need to accept the limitations of data collection that is done in routine medical care. The innovation provided by the application of RASSA is based on a few independent factors with clinical sense identified and easily recorded in health databases, combined in a risk score with high discriminatory capacity. Therefore, our RASSA score model needs to be validated with large data and health systems, in different populations and periods, in order to assure its reliability by external validation. This would be especially important considering that predicting suicide risk from data records relies largely on the clinical judgment and treatment decisions of clinicians, and do not typically reflect important social risk factors such as job loss and relationship disruption [7]. Nevertheless, our results showed the robustness, high specificity and sensitivity, and easy management of our RASSA model. Therefore, we can consider this study as a pilot of a further assessment score to evaluate the risk of suicide attempt that can be applied to different populations and situations.

\section{Conclusions}

Here we present a new approach for risk assessment of suicide attempt among psychiatric population based on clinical records. The score model RASSA identified thirteen social and health markers to estimate the risk of suicide attempt. Its accuracy and easy implementation may provide suggestions for treatment decisions in favour or against adjusting psychotropic medication or changing treatment focus.

\section{List Of Abbreviations}

AUC, area under de curve

BSI, Beck's Scale for Suicide Ideation

$\mathrm{Cl}$, Confidence interval 
DMS-5, Diagnostic and Statistical Manual of Mental Disorders, $5^{\text {th }}$ Edition

HED, hospital's emergency department

ICD-11, International Classification of Diseases, Eleventh Revision

IQR, Interquartile range

MAOI, monoamine oxidase inhibitors

MCMI-IV Millon Clinical Multiaxial Inventory, Fourth Edition

MDE, major depression episode

NPV, negative predictive values

OR, Odds ratio

PPV, positive predictive values

RASSA, risk assessment score of suicide attempt

ROC, Receiver-operating characteristic

SIS, Suicide Intent Scale

SSRI, Selective serotonin reuptake inhibitor

\section{Declarations}

Ethics approval and consent to participate

Study protocol was approved by the Clinical Research Committee at Complejo Asistencial Universitario of Palencia on November $4^{\text {th }}, 2014$.

\section{Consent for publication}

Not applicable.

\section{Availability of data and materials}

Data sharing is not applicable to this article.

\section{Competing interest}

The authors declare that they have no competing interests to declare with respect to the research, authorship, and/or publication of this article. 


\section{Funding}

This research did not receive any specific grants from funding agencies in the public, corporate, or not-forprofit sectors.

\section{Authors' contributions}

All authors were equally involved in the preparation of this manuscript. All authors have read and approved the final manuscript.

\section{Acknowledgements}

We are grateful to the personnel from the Brief Hospitalization Unit of Psychiatry at the Complejo Asistencial Universitario de Palencia- Hospital San Telmo (Palencia, Spain).

\section{References}

1. Harris IM, Beese S, Moore D. Predicting repeated self-harm or suicide in adolescents and young adults using risk assessment scales/tools: A systematic review protocol. Syst Rev. 2019;8(87):1-6.

2. Quinlivan L, Steeg S, Elvidge J, Nowland R, Davies L, Hawton K, et al. Risk assessment scales to predict risk of hospital treated repeat self-harm: A cost-effectiveness modelling analysis. J Affec Disord. 2019;249:208-15.

3. Rice SM, Ogrodniczuk JS, Kealy D, Seidler ZE, Dhillon HM, Oliffe JL. Validity of the Male Depression Risk Scale in a representative Canadian sample: sensitivity and specificity in identifying men with recent suicide attempt. J Ment Health. 2019;28(2):132-40.

4. Sequeira L, Strudwick G, Bailey SM, De Luca V, Wiljer D, Strauss J. Factors influencing suicide risk assessment clinical practice: protocol for a scoping review. BMJ Open. 2019;9(2):e026566.

5. Hughes CW. Objective assessment of suicide risk: significant improvements in assessment, classification, and prediction. Am J Psychiatry. 2011;168(12):1233-4.

6. Torous J, Walker R. Leveraging Digital Health and Machine Learning Toward Reducing Suicide-From Panacea to Practical Tool. JAMA Psychiatry. 2019;76(10):999-1000.

7. Simon GE, Johnson E, Lawrence JM, Rossom R C, Ahmedani B, Lynch FL, et al. Predicting suicide attempts and suicide deaths following outpatient visits using electronic health records. Am J Psychiatry. 2018;175(10):951-60.

8. Stefansson J, Nordström P, Jokinen J. Suicide Intent Scale in the prediction of suicide. J Affect Disord. 2012;136(1-2):167-71.

9. Wilkinson P, Kelvin R, Roberts C, Dubicka B, Goodyer I. Clinical and psychosocial predictors of suicide attempts and no suicidal self-injury in the Adolescent Depression Antidepressants and Psychotherapy Trial (ADAPT). Am J Psychiatry. 2011;168(5):495-501.

10. Holder SD, Wayhs A. Schizophrenia. Am Fam Physician. 2014;90(11):775-82. 
11. Brodsky BS, Malone KM, Ellis SP, Dulit RA, Mann JJ. Characteristics of borderline personality disorder associated with suicidal behaviour. Am J Psychiatry. 1997;154(12):1715-19.

12. Hayes JF, Pitman A, Marston L, Walters K, Geddes JR, King M, et al. Self-harm, Unintentional Injury, and suicide in bipolar disorder during maintenance mood stabilizer treatment: A UK populationbased electronic health records study. JAMA Psychiatry. 2016;73(6):630-7.

13. Holma KM, Melartin TK, Haukka,J, Holma IA, Sokero TP, Isometsä E. Incidence and predictors of suicide attempts in DSM-IV major depressive disorder: A five-year prospective study. Am J Psychiatry. 2010;167(7):801-8.

14. Stübner S, Grohmann R, Greil W, Zhang X, Müller-Oerlinghausen B, Bleich S, et al. Suicidal Ideation and Suicidal Behavior as Rare Adverse Events of Antidepressant Medication: Current Report from the AMSP Multicenter Drug Safety Surveillance Project. Int J Neuropsychopharmacol. 2018;21(9):81421.

15. Christiansen E, Agerbo E, Bilenberg N, Stenager E. SSRIs and risk of suicide attempts in young people - A Danish observational register-based historical cohort study, using propensity score. Nord J Psychiatry. 2016;70(3):167-75.

16. Methling M, Krumbiegel F, Hartwig S, Parr MK, Tsokos M. Toxicological findings in suicides frequency of antidepressant and antipsychotic substances. Forensic Sci, Med Patho. 2019;15(1): 23-30.

17. Näslund J, Hieronymus F, Lisinski A, Nilsson S, Eriksson E. Effects of selective serotonin reuptake inhibitors on rating-scale-assessed suicidality in adults with depression. $\mathrm{Br} \mathrm{J}$ Psychiatry. 2018;212(3):148-54.

18. Gürhan N, Beşer NG, Polat Ü, Koç M. Suicide Risk and Depression in Individuals with Chronic Illness. Community Ment Health J. 2019;55(5):840-8.

19. Keaton SA, Madaj ZB, Heilman P, Smart LA, Grit J, Gibbons R, et al. An inflammatory profile linked to increased suicide risk. J Affect Disord. 2019;247: 57-65.

20. O'Neill S, Graham B, Ennis E. Prescribed pain and mental health medication prior to suicide: A population based case control study. J Affect Disord. 2019;246:195-200.

21. Steele IH, Thrower N, Noroian P, Saleh FM. Understanding Suicide Across the Lifespan: A United States Perspective of Suicide Risk Factors, Assessment \& Management. J Forensic Sci. 2019;63(1):162-71.

22. Levey DF, Polimanti R, Cheng Z, Zhou H, Nuñez YZ, Jain S, et al. Genetic associations with suicide attempt severity and genetic overlap with major depression. Transl Psychiatry. 2019;9(1):22.

23. Belsley DA, Kuh E, Welsh RE. Regression Diagnostics: Identifying Influential Data and Sources of Collinearity. New York: John Wiley \& Sons; 2005.

24. Barak-Corren Y, Castro VM, Javitt S, Hoffnagle AG, Dai Y, Perlis RH, et al. Predicting suicidal behaviour from longitudinal electronic health records. Am J Psychiatry. 2017;174(2):154-62.

25. Hitchcock LS, Ferrell BR, McCaffery M. The experience of chronic non-malignant pain. J Pain Symptom Manage. 1994;9(5):312-8. 
26. Cheatle MD. Depression, chronic pain, and suicide by overdose: on the edge. Pain Med. 2011;12(2):S34-8.

27. Glenn CR, Bagge CL, Osman A. Unique associations between borderline personality disorder features and suicide ideation and attempts in adolescents. J Pers Disord. 2013;27(5):604-16.

28. Muehlenkamp JJ, Ertelt TW, Miller AL, Claes L. Borderline personality symptoms differentiate nonsuicidal and suicidal self-injury in ethnically diverse adolescent outpatients. J Child Psychol Psychiatry. 2011;52(2):148-55.

29. Autry AE, Adachi M, Nosyreva E, Na ES, Los MF, Cheng PF, et al. NMDA Receptor Blockade at Rest Triggers Rapid Behavioural Antidepressant Responses. Nature. 2011;475(7354):91-5.

30. Bhatt M, Perera S, Zielinski L, Eisen RB, Yeung S, El-Sheikh W, et al. Profile of suicide attempts and risk factors among psychiatric patients: A case-control study. PloS One. 2018;13(2):e0192998.

31. Sher L, Kahn RS. Suicide in Schizophrenia: An Educational Overview. Medicina (Kaunas). 2019;55(7):361.

32. Healy D, Aldred G. Antidepressant drug use \& the risk of suicide. Int Rev Psychiatry. 2005;17(3):16372.

33. Delforterie MJ, Lynskey M, Huizink AC, Creemers HE, Grant JD, Few LR, et al. The relationship between cannabis involvement and suicidal thoughts and behaviors. Drug Alcohol Depend. 2015;150:98-104.

34. Jung M. The relationship between alcohol abuse and suicide risk according to smoking status: A cross-sectional study. J Affect Disord. 2019:244:164-70.

35. Yaworski D, Robinson J, Sareen J, Bolton JM. The relation between nicotine dependence and suicide attempts in the general population. Can J Psychiatry. 2011;56(3):161-70.

36. Kessler RC, Berglund PA, Borges G, Castilla-Puentes RC, Glantz MD, Jaeger S, et al. Smoking and suicidal behaviours in the national comorbidity survey: Replication. J Nerv Ment Dis. 2007;195(5):369-77.

37. Kessler RC, Borges G, Sampson N, Miller M, Nock M. The association between smoking and subsequent suicide related outcomes in the National Comorbidity Survey panel sample. Mol Psychiatry. 2009;14(12):1132-42.

38. Fernández Guijarro S, Miguel García C, Pomarol-Clotet E, Egea López EN, Burjales Martí MD, Rigol Cuadra MA. Metabolic Syndrome Screening in People With Severe Mental Illness: Results From Two Spanish Community Mental Health Centers. J Am Psychiatr Nurses Assoc. 2020;26(2):162-71.

39. Mallet J, Le Strat Y, Schürhoff F, Mazer N, Portalier C, Andrianarisoa M, et al. Tobacco smoking is associated with antipsychotic medication, physical aggressiveness, and alcohol use disorder in schizophrenia: results from the FACE-SZ national cohort. Eur Arch Psychiatry Clin Neurosci. 2019;269(4):449-57.

40. Oquendo MA, Turret J, Grunebaum MF, Burke AK, Poh E, Stevenson E, et al. Sex differences in clinical predictors of depression: A prospective study. J Affect Disord. 2013;150(3):1179-83. 
41. Vijayakumar L, Lamech N. Suicide and Suicidal Behavior in Women. In: Prabha Chandra, Helen Herrman, Jane Fisher, Anita Riecher-Rössler, editors. Mental Health and Illness of Women. Singapore: Springer Singapore; 2019. p. 35-56.

42. Vijayakumar L. Suicide in women. Indian J Psychiatry. 2015;57(Suppl 2):S233-8.

43. Fourestié $V$, de Lignières $B$, Roudot-Thoraval F, Fulli-Lemaire I, Cremniter $D$, Nahoul $K$, et al. Suicide attempts in hypo-oestrogenic phases of the menstrual cycle. Lancet. 1986;2(8520):1357-60.

\section{Tables}

Table 1. Main characteristics of case and control subjects in the study (2000-2014). 


\begin{tabular}{|c|c|c|c|c|c|}
\hline & & $\begin{array}{l}\text { ontrol Group } \\
(\mathrm{N}=1,120)\end{array}$ & & $\begin{array}{l}\text { Case Group } \\
(\mathrm{N}=560)\end{array}$ & \\
\hline Characteristics & $\mathrm{N}$ & Median [Q1-Q3] & $\mathrm{N}$ & Median [Q1-Q3] & $\mathrm{p}$-value \\
\hline Age (years) & 1120 & $34[27-46]$ & 560 & 34 [26-45] & 0.460 \\
\hline \multirow[t]{2}{*}{ Visits to HED } & 385 & $2[1-2]$ & 31 & $31[1-1]$ & 0.000 \\
\hline & $\mathrm{N}$ & $\%$ & $\mathrm{~N}$ & $\%$ & p-value \\
\hline Woman (sex) & 714 & 63.8 & 379 & 67.7 & 0.111 \\
\hline Single (marital status) & 373 & 33.3 & 277 & 49.5 & 0.000 \\
\hline Previous suicide attempt (Yes) & 0 & 0.0 & 327 & 58.4 & 0.000 \\
\hline Family history of suicide (Yes) & 95 & 8.5 & 134 & 23.9 & 0.000 \\
\hline Depression (main disorder) & 200 & 17.9 & 276 & 49.3 & 0.000 \\
\hline MDE-3 (Yes) & 331 & 29.6 & 263 & 47.0 & 0.000 \\
\hline Personality disorder (Yes) & 100 & 8.9 & 560 & 100 & 0.000 \\
\hline $\begin{array}{l}\text { Attending a psychotherapy } \\
\text { program }\end{array}$ & 304 & 27.1 & 151 & 27.0 & 0.938 \\
\hline Non-psychiatric comorbidity (Yes) & 647 & 57.8 & 495 & 88.4 & 0.000 \\
\hline Benzodiazepines (Yes) & 797 & 71.2 & 399 & 71.3 & 0.970 \\
\hline Antidepressants (Yes) & 730 & 65.2 & 413 & 73.8 & 0.000 \\
\hline SSRIs (Yes) & 416 & 37.1 & 301 & 53.8 & 0.000 \\
\hline Antipsychotics (Yes) & 698 & 62.3 & 146 & 26.1 & 0.000 \\
\hline Anticonvulsants (Yes) & 342 & 30.5 & 115 & 20.5 & 0.000 \\
\hline Opioid analgesics (Yes) & 47 & 4.2 & 28 & 5.0 & 0.452 \\
\hline Non-opioid analgesics (Yes) & 268 & 23.9 & 336 & 60.0 & 0.000 \\
\hline Tobacco (Yes) & 663 & 59.2 & 249 & 44.5 & 0.000 \\
\hline Alcohol (Yes) & 340 & 30.4 & 203 & 36.3 & 0.015 \\
\hline Cannabis (Yes) & 135 & 12.1 & 72 & 12.9 & 0.637 \\
\hline Cocaine (Yes) & 18 & 1.6 & 27 & 4.8 & 0.000 \\
\hline Heroin (Yes) & 6 & 0.5 & 2 & 0.4 & 0.616 \\
\hline Amphetamine (Yes) & 6 & 0.5 & 9 & 1.6 & 0.028 \\
\hline
\end{tabular}


MDE-3: refers to major depression episode in the three months prior to suicide attempt. 'Benzodiazepines', 'antidepressants', 'opioid analgesics', 'non-opioid analgesics', 'anticonvulsants' and 'SSRIs' refer to drug treatment. 'Tobacco', 'cannabis' and 'cocaine' refer to substance use disorder. 'Visits to HED' refers to visits to the Hospital's emergency department.

Table 2. Antidepressants used by psychiatric patients in the three months prior to suicide attempt (2000-2014). 


\begin{tabular}{|c|c|c|c|c|c|c|}
\hline \multirow{3}{*}{$\begin{array}{l}\text { Selective serotonin } \\
\text { reuptake inhibitors (SSRIs) }\end{array}$} & \multicolumn{2}{|c|}{$\begin{array}{l}\text { Control Group } \\
\qquad(\mathrm{N}=1,120)\end{array}$} & \multicolumn{2}{|c|}{$\begin{array}{l}\text { Case Group } \\
\qquad(\mathrm{N}=560)\end{array}$} & \multicolumn{2}{|c|}{$\begin{array}{c}\text { Total } \\
(\mathrm{N}=1,680)\end{array}$} \\
\hline & $\mathrm{N}$ & $\%$ & $\mathrm{~N}$ & $\%$ & $\mathrm{~N}$ & $\%$ \\
\hline & & & & & & \\
\hline Escitalopram & 54 & 4.8 & 67 & 12.0 & 121 & 7.2 \\
\hline Venlafaxine & 126 & 11.3 & 45 & 8.0 & 171 & 10.2 \\
\hline Paroxetine & 27 & 2.4 & 44 & 7.9 & 71 & 4.2 \\
\hline Sertraline & 19 & 1.7 & 38 & 6.8 & 57 & 3.4 \\
\hline Citalopram & 46 & 4.1 & 35 & 6.3 & 81 & 4.8 \\
\hline Fluvoxamine & 29 & 2.6 & 34 & 6.1 & 63 & 3.8 \\
\hline Fluoxetine & 54 & 4.8 & 25 & 4.5 & 79 & 4.7 \\
\hline Trazodone & 61 & 5.4 & 13 & 2.3 & 74 & 4.4 \\
\hline Non-SSRIs & $\mathrm{N}$ & $\%$ & $\mathrm{~N}$ & $\%$ & $\mathrm{~N}$ & $\%$ \\
\hline Imipramine (Tricyclics) & 29 & 2.6 & 20 & 3.6 & 49 & 2.9 \\
\hline Nortriptyline (Tricyclics) & 54 & 4.8 & 12 & 2.1 & 66 & 3.9 \\
\hline Agomelatine (Others) & 30 & 2.7 & 11 & 2.0 & 41 & 2.4 \\
\hline Bupropion (Others) & 27 & 2.4 & 11 & 2.0 & 38 & 2.3 \\
\hline Tranylcypromine (MAOI) & 13 & 1.2 & 11 & 2.0 & 24 & 1.4 \\
\hline Mirtazapine (Others) & 30 & 2.7 & 8 & 1.4 & 38 & 2.3 \\
\hline Clomipramine (Tricyclics) & 25 & 2.2 & 8 & 1.4 & 33 & 2.0 \\
\hline Reboxetine (Others) & 30 & 2.7 & 7 & 1.3 & 37 & 2.2 \\
\hline Amitriptyline (Tricyclics) & 27 & 2.4 & 7 & 1.3 & 34 & 2.0 \\
\hline Moclobemide (MAOI) & 19 & 1.7 & 7 & 1.3 & 26 & 1.5 \\
\hline Duloxetine (Others) & 30 & 2.7 & 6 & 1.1 & 36 & 2.1 \\
\hline Venlafaxine (Others) & 26 & 2.3 & 5 & 0.9 & 31 & 1.8 \\
\hline Non-antidepressants & 364 & 32.5 & 146 & 26.1 & 510 & 30.4 \\
\hline
\end{tabular}

MAOI: monoamine oxidase inhibitors; SSRIs: selective serotonin reuptake inhibitors. 
Table 3. Logistic regression analysis and risk factors for suicide attempt in psychiatric patients $(1,680)$ by retrospective analysis of their health records (2000-2014).

\begin{tabular}{lccccccccc}
\hline Variables & & & & & & & \multicolumn{2}{c}{$95 \%$ C.I. } \\
\cline { 6 - 9 } (Risk factors) & $\mathrm{b}$ & S.E. & Wald & d.f. & $\mathrm{p}$ & $\mathrm{OR}$ & $\mathrm{L}$ & $\mathrm{U}$ \\
\hline Female (sex) & 0.633 & 0.148 & 18.204 & 1 & 0.000 & 1.883 & 1.408 & 2.518 \\
Single (marital status) & 0.715 & 0.139 & 26.458 & 1 & 0.000 & 2.044 & 1.557 & 2.685 \\
\hline Family history of suicide (Yes) & 1.410 & 0.197 & 51.275 & 1 & 0.000 & 4.097 & 2.785 & 6.026 \\
\hline Antipsychotics (No) & 1.628 & 0.143 & 128.697 & 1 & 0.000 & 5.093 & 3.844 & 6.747 \\
\hline Opioid analgesics (No) & 0.877 & 0.308 & 8.132 & 1 & 0.004 & 2.405 & 1.316 & 4.395 \\
\hline Non-opioid analgesics (Yes) & 1.377 & 0.141 & 96.051 & 1 & 0,000 & 3.964 & 3.010 & 5.222 \\
\hline Tobacco (No) & 0.543 & 0.147 & 13.653 & 1 & 0.000 & 1.721 & 1.290 & 2.295 \\
\hline Cannabis (Yes) & 0.729 & 0.220 & 10.975 & 1 & 0.001 & 2.074 & 1.347 & 3.193 \\
\hline Cocaine (Yes) & 1.299 & 0.422 & 9.468 & 1 & 0.002 & 3.667 & 1.603 & 8.388 \\
\hline Depression (Yes) & 1.328 & 0.150 & 78.799 & 1 & 0.000 & 3.774 & 2.815 & 5.060 \\
\hline SSRIs (Yes) & 0.514 & 0.139 & 13.592 & 1 & 0.000 & 1.672 & 1.272 & 2.198 \\
\hline Non-psychiatric comorbidity (Yes) & 1.621 & 0.177 & 84.091 & 1 & 0.000 & 5.057 & 3.577 & 7.151 \\
\hline MDE-3 (Yes) & 0.649 & 0.142 & 21.050 & 1 & 0.000 & 1.915 & 1.451 & 2.527 \\
\hline
\end{tabular}

b: regression coefficient; C.I.: confidence interval; d.f.: degrees of freedom; L: lower limit; P: probability; S.E.: standard error; U: upper limit. Wald: Wald statistics. 'Tobacco', 'cannabis' and 'cocaine' refer to substance use disorder. Depression: main disorder. Comorbidity: somatic disorder. MDE-3: major depression episode in the three months prior to the attempt.

Table 4. Performance characteristics of RASSA at various cut-off points for evaluation of suicide attempt score in psychiatric patients $(1,680)$ using information from their health records (2000-2014). 


\begin{tabular}{lcccccccc}
\hline $\begin{array}{l}\text { RASSA Score } \\
\text { Cut-Points }\end{array}$ & TP & TN & FP & FN & Sen (\%) & Spe (\%) & PPV (\%) & NPV (\%) \\
\hline 15 & 549 & 449 & 671 & 11 & 98.0 & 40.1 & 45.0 & 97.6 \\
20 & 513 & 801 & 319 & 47 & 91.6 & 71.5 & 61.7 & 94.5 \\
25 & 439 & 1033 & 87 & 121 & 78.4 & 92.2 & 83.5 & 89.5 \\
30 & 329 & 1098 & 22 & 231 & 58.8 & 98.0 & 93.7 & 82.6 \\
35 & 219 & 1118 & 2 & 341 & 39.1 & 99.8 & 99.1 & 76.6 \\
40 & 132 & 1120 & 0 & 428 & 23.6 & 100 & 100 & 72.4 \\
\hline
\end{tabular}

$\mathrm{TP}=$ true positives; $\mathrm{TN}=$ true negatives; $\mathrm{FP}=$ false positives; $\mathrm{FN}=$ false negatives; $\mathrm{Sen}(\%)=$ Sensitivity; Spe $(\%)=$ Specificity; PPV = positive predicted value; NPV = negative predictive value.

\section{Figures}

\begin{tabular}{ll}
\hline Condition & Points \\
\hline Gender (female) & 2 \\
Marital status (single) & 3 \\
Family history of suicide (yes) & 5 \\
Antipsychotic drugs (no) & 6 \\
Opioid analgesics (no) & 3 \\
Non-opioid analgesics (yes) & 5 \\
Tobacco dependence (no) & 2 \\
Cannabis abuse/dependence (yes) & 3 \\
Cocaine abuse/dependence (yes) & 5 \\
Depression (yes) & 5 \\
SSRIs intake (yes) & 2 \\
Comorbidity (yes) & 6 \\
Depression episode in the las 3 months (yes) & 3
\end{tabular}

RASSA Score $=\Sigma$ Points

If previous attempt: RASSA Score $=1.4 \times \mathbf{\Sigma}$ Points

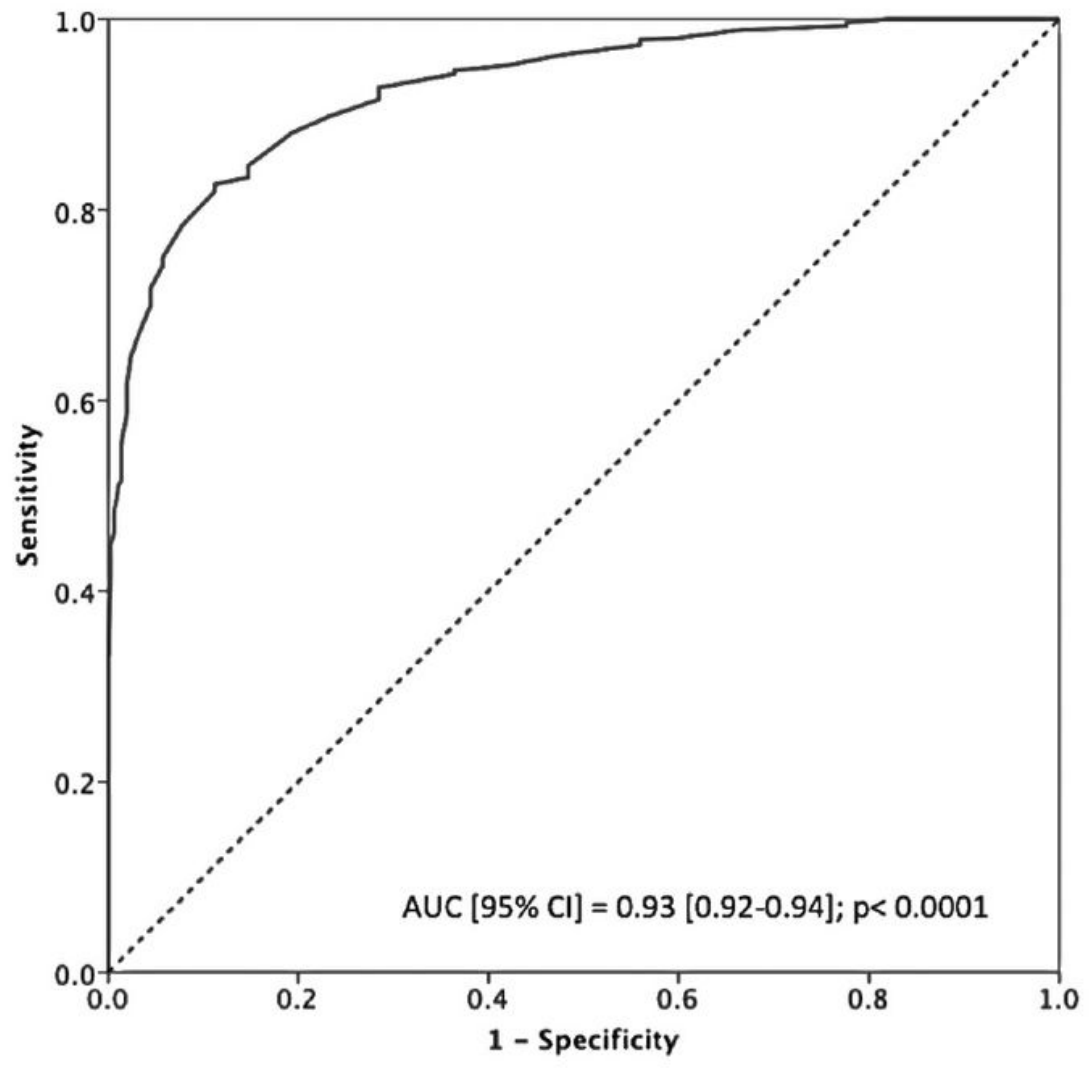

Figure 1 
Scale with scores for the risk assessment and suicide attempt prediction in psychiatric patients (left) and Receiving Operating Characteristic (ROC) curve for the scale in suicide prediction (right). Risk factor score is added in the count when the patient is assigned to the specified category of the variable. SSRIs: selective serotonin reuptake inhibitor. 